\title{
Self-consistent chaos and Arnold diffusion in a cyclotron-maser wave-particle system
}

\author{
R. Pakter, F. O. Couto, and F. B. Rizzato \\ Instituto de Física, Universidade Federal do Rio Grande do Sul, Caixa Postal 15051, \\ 91501-970 Porto Alegre, Rio Grande do Sul, Brazil
}

(Received 16 March 1995; revised manuscript received 12 May 1995)

\begin{abstract}
In this work, we search for the presence of chaos in a self-consistent model for the cyclotronresonance maser accelerator. Two characteristic regimes are identified. If the initial action variable of the accelerating particles is small, rapid phase bunching occurs and the particle population is condensed into a single macroparticle in phase space. As a result, this low-dimensional state is predominantly regular. For larger energies, on the other hand, there is no macroparticle formation. The system is high dimensional, and chaos is found. Arnold diffusion appears to occur in these chaotic states.
\end{abstract}

PACS number(s): 05.45.+b, 41.75.-i

\section{INTRODUCTION}

A promising configuration for laser acceleration of charged particles is the so-called cyclotron-resonance maser accelerator (CRMA) [1], where a coherent electromagnetic wave may transfer a large amount of energy to a beam of electrons gyrating in a guide magnetic field. This large amount of transferred energy takes place because of the autoresonance mechanism [2-5] whereby an initial wave-particle synchronism may be self-sustained throughout the accelerating period.

The good quality of the autoresonance mechanism and the resulting high efficiency of the CRMA depend on some conditions that must be observed, two of which are currently considered of some particular relevance. One of them demands that the maser wave be dispersionless. This is hard to satisfy experimentally because the presence of waveguide and finite confining systems always introduce some dispersion in the system. A number of recent works shows, however, how the problem can be circumvented. Chen [6], for instance, uses tapered magnetic fields to maintain the resonance that would be otherwise destroyed by dispersion. Alternatively, Pakter et al. [7] make use of a small mismatch between the Doppler-shifted cyclotron and wave frequencies to completely compensate the dispersive effects.

Another condition to be satisfied is that, in an actionangle representation, the initial action variables of the electrons be very small. If this is the case, one can show that the entire beam is rapidly bunched in phase space, behaving as a single macroparticle $[1,7]$. The coherent beam thus formed is afterwards accelerated to the maximum available electromagnetic energy. Note that in this case the complete dynamical system would be reduced to a few degrees of freedom-those describing the single macroparticle plus those describing the maser field. In fact, in this case the system turns out to have only one effective degree of freedom and therefore is completely integrable [8]. If, on the other hand, the control over the injected beam is poor, the low-action condition is unlikely to be satisfied. Then one will end up with a very incoherent beam whose description will require all degrees of freedom of the complete system. In view of the effective multidimensionality, the associated dynamics would be almost certainly chaotic.

Chaos in CRMA's is a possibility recently investigated by Pakter et al. [9]. In the model analyzed, the amplitude of the maser field undergoes externally produced slow modulations, which induces the appearance of series of period doubling and inverse saddle-node bifurcations leading to chaos. Amplitude variations can be easily generated if the maser wave is modulational unstable, as in the case of various wave-particle accelerating systems like the ones found in the magnetosphere of pulsars, for instance [10]. However, it is not clear whether this kind of process can be indeed self-consistently generated in a CRMA.

The purpose of this paper is therefore to search for the presence of self-consistent chaos in CRMA's. In our procedures, low-energy beams with initially homogeneous gyrophase distributions are released under the influence of a strong-amplitude maser field, and the appropriate Lyapunov exponents measuring the divergence of the trajectories of the entire dynamical system are examined; the presence of exponential orbital divergence is taken as the signature of the existence of chaotic states. It turns out that we do find chaos in the system. Moreover, unlike some typical cases of wave-particle systems, we find that chaotic activity is large not only near the separatrix of the system, but also near the elliptic fixed points. We finally propose a single low-dimensional model that comprises some of the basic features observed. Our results are shown to be related to those recently obtained by Boozer on Arnold diffusion [11].

The paper is therefore organized as follows. In Sec. II we introduce the model and the basic equations to be used; in Sec. III we perform some exploratory simulations; in Sec. IV we search for the presence of positive Lyapunov exponents; in Sec. V we make use of a lowdimensional model to explain the results obtained in Sec. IV, and in Sec. VI we conclude the work. 


\section{MODEL AND BASIC EQUATIONS}

In the model, we consider an electron beam and a circularly polarized electromagnetic wave, co-propagating along the homogeneously magnetized $z$ axis of the chosen reference frame. With the appropriate set of normalizations and canonical transformations (see Pakter et al. [7] for details), it becomes possible to write down the complete set of equations governing the self-consistent dynamics of particles plus maser field in the form

$$
\begin{gathered}
d_{t} I_{j}=-\partial_{\phi_{j}} H \\
d_{t} \phi_{j}=\partial_{I_{j}} H \\
d_{t} \rho=-\partial_{\sigma} H
\end{gathered}
$$

and

$$
d_{t} \sigma=\partial_{\rho} H
$$

with $j=1,2,3, \ldots, N, d_{t} \equiv \frac{d}{d t}, \partial_{-} \equiv \frac{\partial}{\partial_{-}}$, and $H=$ $\sum_{j} H_{j}$, with

$$
H_{j}=-\omega I_{j}+\sqrt{1+2 I_{j}+\left(\omega I_{j}\right)^{2}+2 \sqrt{2 I_{j} \lambda \rho} \cos \left(\phi_{j}+\sigma / N\right)+\lambda \rho} .
$$

The quantity $N$ is the number of particles used in the simulations or, more precisely, the number of macroparticles used in the simulations - we typically take $N=$ 500 - and the index $j$ labels the particles. Variables $\rho(t)$ and $\sigma(t) / N$ are, respectively, the time-dependent amplitude and phase of the slowly modulated high-frequency carrier wave whose normalized vector potential is written in complete form as

$$
\begin{aligned}
\mathbf{A}= & \sqrt{\lambda \rho}\{-\cos [\omega(z-t)+\sigma / N] \hat{\mathbf{x}} \\
& +\sin [\omega(z-t)+\sigma / N] \hat{\mathbf{y}}\} .
\end{aligned}
$$

The system is dispersionless with $(\omega, \omega \hat{\mathbf{z}})$ standing for the dimensionless wave frequency and wave vector. Variables $I_{j}$ and $\phi_{j}$ are, respectively, the action and gyrophase (actually the gyrophase as measured relatively to the wave phase) of the $j$ th particle. Finally, $\lambda$ satisfies $\lambda \ll 1$ and is proportional to the tenuous particle density of the system.

We point out that in obtaining Eqs. (1)-(5) we made use of the assumption $P_{z, j}(t=0)=0$, where $P_{z, j}(t=0)$ is the injected (or initial) value of the $z$ component of the canonical momentum. Although this simplifying condition is inappropriate for laboratory CRMA's, where $P_{z, j}(t=0)$ is larger [12], we feel that the general results we are about to obtain here with $P_{z}=0$ would not suffer dramatic changes had we used $P_{z} \neq 0$ instead. In any case, cyclotron acceleration is relevant matter in other systems, like the Earth's or pulsars' magnetospheres, where $P_{z}(t=0)$ is arbitrary.

As can be seen from the equations above, the dynamical equations for any $i$ particle can be obtained from a single particle Hamiltonian which depends only on the $i$ coordinates and on the maser variables,

$$
H_{i}=H_{i}\left(I_{i}, \phi_{i}, \lambda \rho, \sigma\right) .
$$

This feature tells us that the interaction among the particles is exclusively mediated by the maser field, i.e., there is no direct particle-particle interaction. This is relevant information, because the basic aspects of the system could be understood if one could somehow obtain a fairly accurate model for the field. Obtaining the accurate model is, of course, no easy task. In principle one has to solve the full problem and then look at the field in order to guess the good model. This is true, but as we shall see, at least for some cases, there are certain basic dynamical properties of the maser which seem to be more or less independent of external influences such as initial conditions and fixed external parameters. We shall make use of these basic properties in order to study the type of chaotic dynamics we shall have at hand.

\section{INITIAL SIMULATIONS ON THE PHASE SPACE}

Let us now numerically solve the set (1)-(4). To do that, let us recall that we shall be injecting monoenergetic beams with homogeneous gyrophase spreads. In concrete terms this means $I_{j}(t=0)=I_{0}$ and $\phi_{j}=(j / N) 2 \pi$ for $j=1,2, \ldots, N$. As for the wave, we consider $\rho(t=$ $0)=\rho_{0}$ and $\sigma(t=0)=0$. Our program of study is to examine the dynamics as a function of $I_{0}$; we shall see that the most interesting aspects of the problem do appear when the initial action is varied. Besides we shall consider henceforth $\rho_{0}=100$ and $\lambda=0.01$ as these values correspond to physically relevant settings [7]. Finally we take $\omega=1 / \sqrt{1+\lambda \rho_{0}}=1 / \sqrt{2}$; it can be shown that otherwise autoresonance is absent [9].

Before embarking in the simulations let us plot some particle trajectories of our system on a common $I, \phi$ phase space, keeping $\rho$ and $\sigma$ constants. This, of course, does not represent an actual physical situation because phase and wave amplitude are not varying selfconsistently. However, this kind of plot provides useful information in what follows. The plot is shown in Fig. 1. Among other characteristics, two are of relevance: (i) there exist two elliptic fixed points located at $\phi=0$ and $\phi=\pi$ and (ii) particles initially located close to $I=0, \phi=\pi / 2$ tend to be lifted almost vertically, gaining therefore a large amount of energy.

If it were not for the finite amount of electromagnetic energy which causes the electromagnetic field to vary selfconsistently, those particles would be accelerated without limits - this is the autoresonance mechanism (we mention that the trajectory at $\phi=\pi / 2$ is exactly vertical, pointing upwards at any value of $I$; if $\omega \neq 1 / \sqrt{1+\lambda \rho_{0}}$ no vertical trajectory is present). As the other limiting effect on autoresonance, wave dispersion, may be compen- 


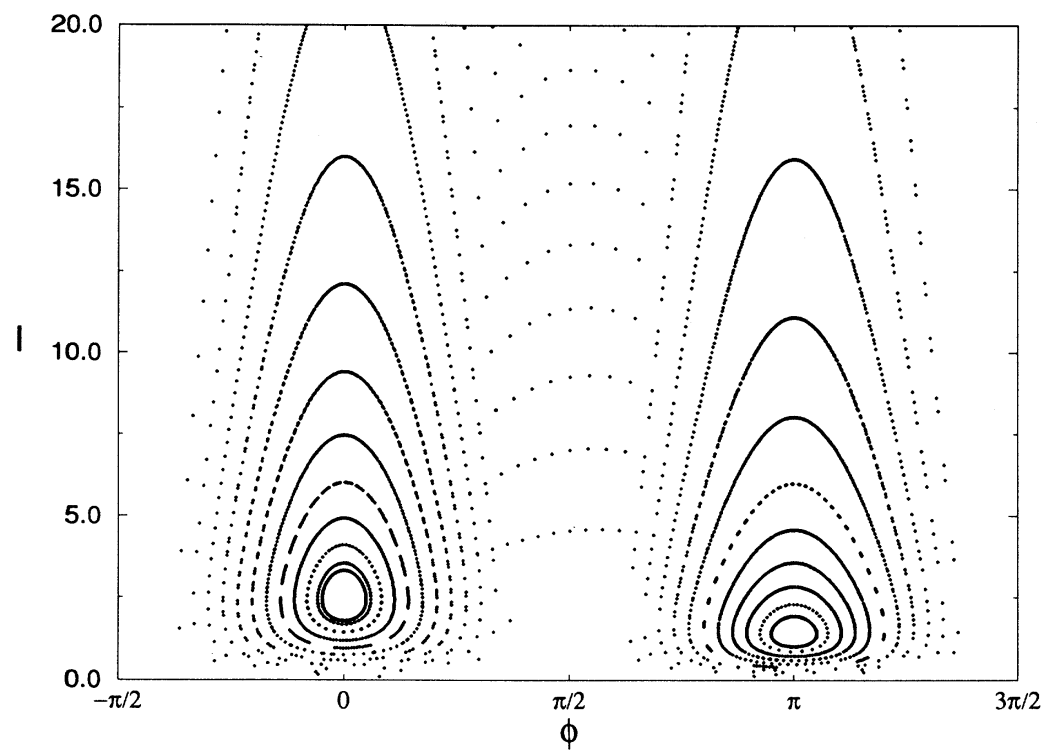

FIG. 1. Phase space for fixed wave amplitude and wave phase. As in all following cases, we take $\lambda=0.01, \rho_{0}=100$, and $\sigma(t=0)=0$. sated with the techniques mentioned in the Introduction, it shall not be further considered here.

To conclude, we quote that calculations performed elsewhere [9] indicate that the gyrofrequency goes to zero as the autoresonance orbit is approached. In that sense, the autoresonance can be thus seen as somewhat equivalent to a separatrix orbit-as a matter of fact, it separates orbits encircling the $\phi=0$ fixed point from those encircling the $\phi=\pi$ fixed point.

\section{A. Bunched states}

Let us consider $I_{0} \ll 1$ initially. Then use of Hamiltonian (5) in the dynamical equations readily shows, as reported in Pakter et al. [7], that a large $1 / \sqrt{I}$ term dominates the right-hand side of Eq. (4), inducing a fast bunching process. The bunching process is such that during a very short period of time particles are rapidly driven into a highly condensed state in phase space whose actions and gyrophases satisfy $I_{j} \sim I_{0}$ and $\phi_{j} \sim \pi / 2$. This bunched group of particles, which can be described in terms of a single macroparticle, is afterwards accelerated to the maximum allowed energy provided by the electromagnetic field. The maximum action $I_{\max }$ attained by the single macroparticle can be calculated with help of the constants of motion derived from Eqs. (1)-(4); it reads $I_{\max }=\rho_{0}$, meaning that when bunching is present, all the available electromagnetic energy can be transferred to particles. One important remark to be made here is that the effective number of degrees of freedom is presently reduced to two. One degree of freedom describes the macroparticle and one degree describes the maser field. Then, as gyrophases and wave phase always appear in the form $\phi+\sigma / N$, further reduction of degrees of freedom becomes possible, and the entire system ends up being described only in terms of one degree of freedom. Thus, as one learns from nonlinear dynamics, the accelerating process in this case is of the regular, and not chaotic, type.

We now examine a snapshot of the particle phase space at $t=11200$ in Fig. 2(a). There, one can clearly see that despite the fact that the initial distribution was homogeneously spread along the $\phi$ axis, the final state is highly condensed-bunching does indeed occur as we have indicated.

Moreover, Fig. 2(b) indicates that the time series for the relevant quantities [we plot $\rho(t)$ vs $t$ ] are periodic, as it should be for a low-dimensional conservative regular system; wave and macroparticle just keep exchanging energy in a regular fashion among themselves.

\section{B. Unbunched states}

Let us now turn to the case where $I_{0}$ is comparable to the unity. The tuning range of cyclotron accelerating systems is small, so that if one fixes the initial wave amplitude as in the former case, $\lambda \rho(t=0)=1$, but considers much larger values of $I_{0}$, coherent acceleration should not be expected to occur. This kind of situation may well represent poorly controlled systems (e.g., the magnetosphere of Earth and pulsars) where a precise wave-particle resonance like the previous one is very unlikely. In this case there is no bunching mechanism and consequently no reduction on the degrees of freedom. The dynamics is therefore expected to reveal some complex, or perhaps chaotic characteristics. Let us first plot a snapshot of the phase space, at $t=11200$, of a distribution which initially had $I_{0}=2$ (besides being homogeneous in gyrophase). Then one has a picture like the one represented in Fig. 3(a). From the picture we conclude that a bunched distribution is indeed no longer formed and that the overall dynamics appears to be irregular.

As the particle distribution ends up by largely spreading itself over the phase space, one expects the waveparticle energy exchange to be much smaller than in the 
former case (if one particle is gaining energy, one is losing, and so on-this is a manifestation of energy equipartition). This is true, as can be seen from Fig. 3(b), where the time series for $\rho(t)$ is shown. After some initial transient where the maser shares its "excess" energy with the particles, it settles down to an average level close to $\rho=60$ plus some small fluctuations. We believe that this is a typical asymptotic state in the case $I_{0} \sim O(1)$.

From the figure one can see that the time scales of the small fluctuations are roughly distributed between a longest, $T_{l}$, and a shortest, $T_{s}$, scale. Moreover, one has approximately $T_{l} \sim 5000$ and $T_{s} / T_{l} \sim 0.1$.

Both scales have been compared with frequencies of typical orbits and we have arrived at the following conclusions. The longest scale is dictated by the remaining wave-particle energy exchange involving particles which move with very small frequencies on the phase space. Those particles are the ones describing the outermost orbits of Fig. 3(a). The frequencies are small because these outermost orbits are very close to autoresonance. From Fig. 1 it is indeed possible to see that the closer an orbit is to autoresonance, the larger is its range.

The shortest scale, on the other hand, comes from the wave-particle interaction involving the bulk of the particle distribution. Particles in the bulk are closer to the elliptic fixed points and describe higher-frequency orbits.

The fluctuational effect is real and not a mere consequence of the graininess introduced by the finite number of macroparticles used in the simulations. Runs were performed with $N=1000$ and $N=1500$ and the results obtained were identical to the ones presented here.

\section{CHAOTIC AND REGULAR STATES}

\section{A. Global chaotic and nonchaotic states}

To search for the presence of chaotic states, we adopt the following procedure. Along with the original par-
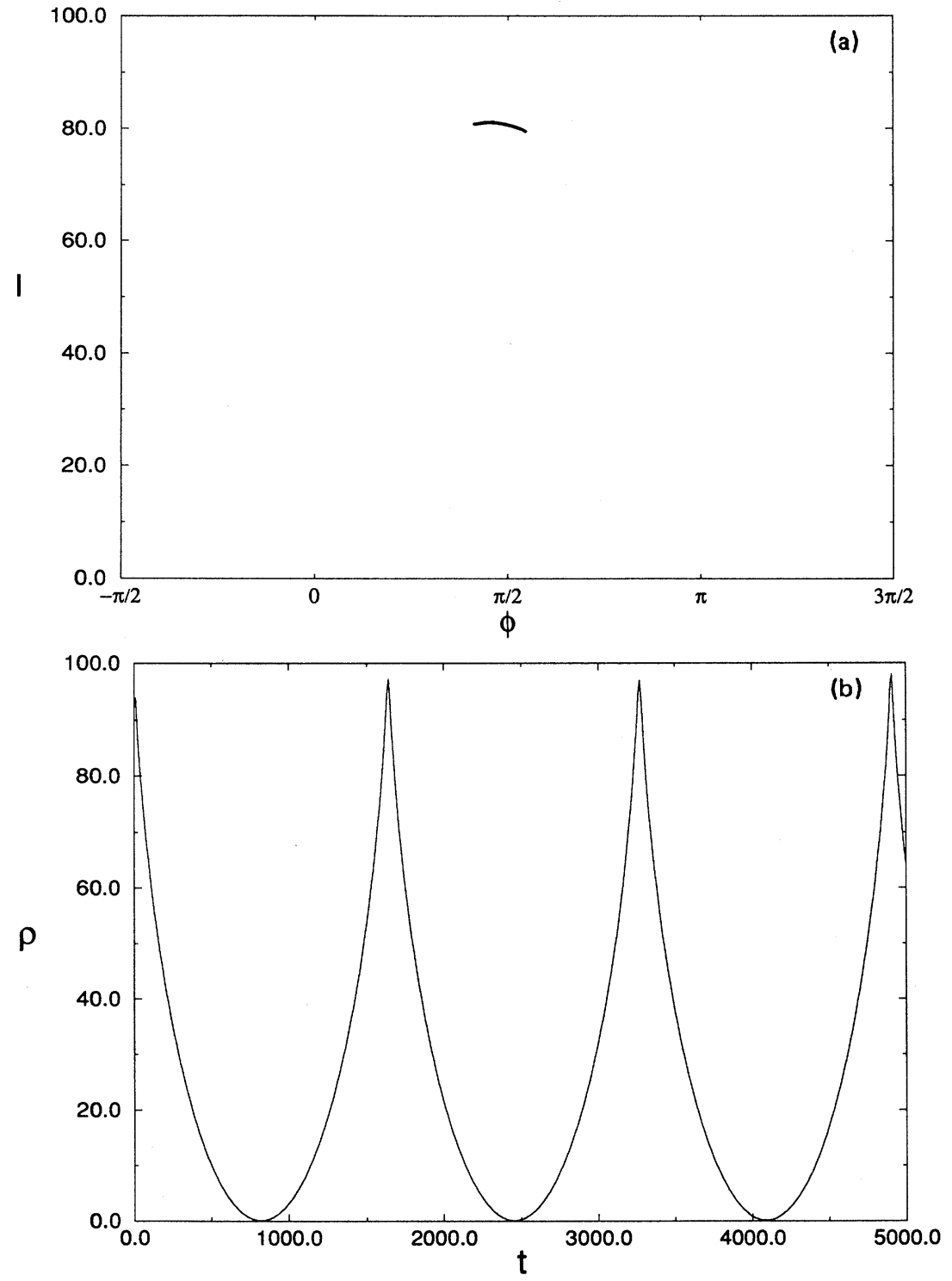

FIG. 2. Self-consistent bunched particle distribution obtained for $I_{0}=0.001$ in (a). The corresponding $\rho(t)$ time series in (b). 
ticle distribution which self-consistently interacts with the maser field, we launch a second distribution with the same number of particles as the original. This second distribution is acted upon by the maser field exactly as the original, but does not react back upon the field. In other words, this second distribution is a test distribution. Particles of the second distribution (which we denote henceforth as the primed distribution) have their initial actions and gyrophases located very close to those of the original distribution according to the rule $I_{j}^{\prime}=I_{j}(1+\delta)$ and $\phi_{j}^{\prime}=\phi_{j}(1+\delta), j=1,2, \ldots, N$, where $\delta$ is a small number, typically $\delta \sim 10^{-10}$. Then, we introduce function $L(t)$ defined by

$L(t) \equiv \ln \left[\sqrt{\sum_{j}\left[I_{j}(t)-I_{j}^{\prime}(t)\right]^{2}+\left[\phi_{j}(t)-\phi_{j}^{\prime}(t)\right]^{2}}\right]$.
Whenever function $L$ behaves as straight line, this is considered as signaling the presence of exponentially diverging trajectories and, consequently, of chaos [8]. Indeed, the linear coefficient of the straight line is formally considered to be the Lyapunov coefficient $\left(\gamma_{L}\right)$ of the original, or unprimed, orbit.

In Fig. 4 we simultaneously plot three $L$ [normalized to $L(t=0)$ ] curves for the three cases so far studied: (a) the self-consistent unbunched case, $I_{0}=2 \sim O(1)$; (b) the self-consistent bunched case, $I_{0}=0.001 \ll 1 ;$ (c) and the case where wave amplitude and phase are artificially kept constant, the one represented in Fig. 1. Function $L(t)$ behaves as a straight line with positive slope only in the first case. In the second case only a very weak orbital divergence, if any, takes place, and in the last case the behavior is definitely logarithmiclike. Following Lichtenberg and Lieberman [8], we point out that this logarithmic behavior is quite typical of multidimensional
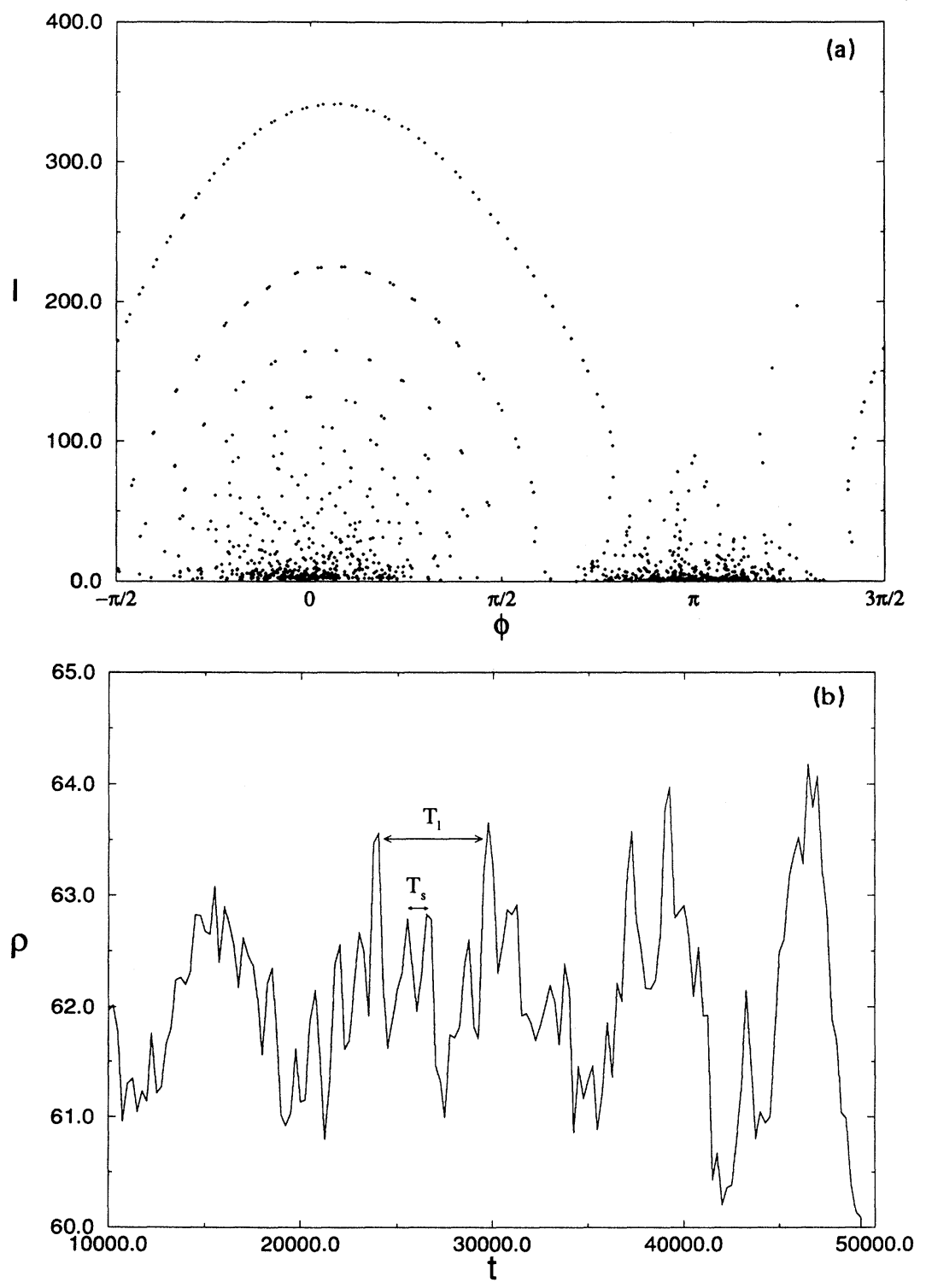

FIG. 3. The same as in Fig. 2, now for the unbunched case obtained with $I_{0}=2$. 


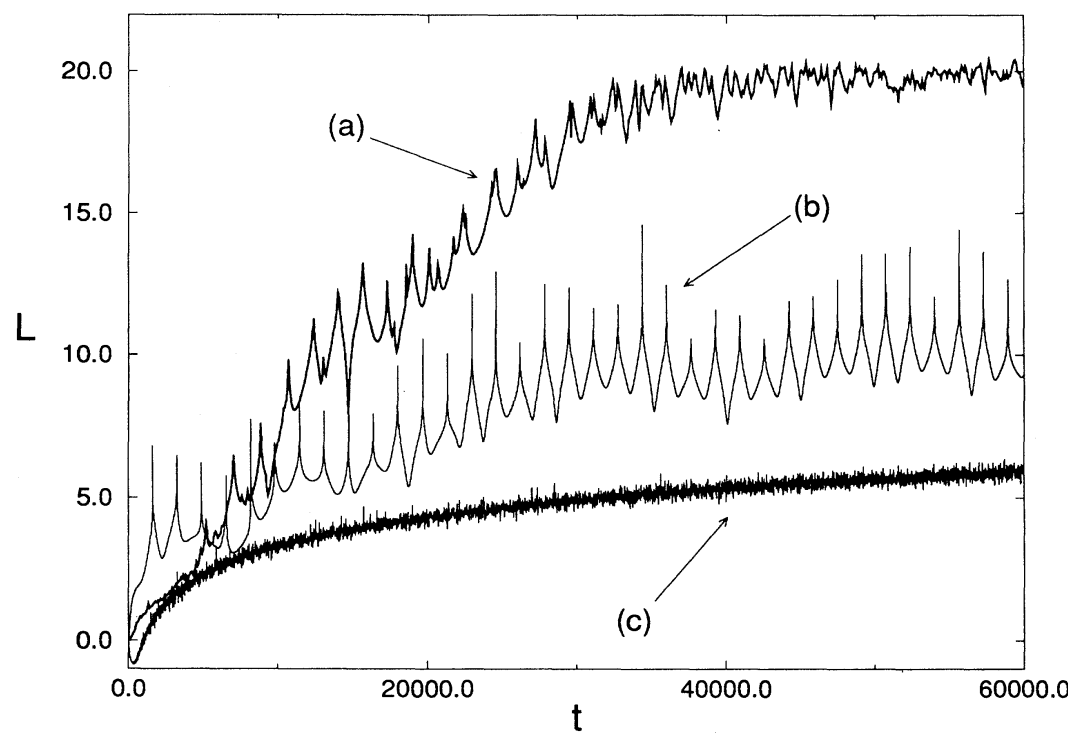

integrable systems. One can also observe the formation of a plateau, in curve (a), reflecting the size scale of the phase space.

Only the first case can be safely said to develop chaos. We have also performed the numerical computation of the Lyapunov coefficient for this chaotic case, according to a known renormalization technique $[8,13]$. The value thus obtained is $\gamma_{L} \sim 7.0 \times 10^{-4}$, which agrees with curve (a) of Fig. (4).

The maser wave is also a dynamical entity of the system and its corresponding dynamical variables, amplitude and phase, should have also been included in the summation defining function $L(t)$. We did that and found no significant difference in the results as compared to the ones obtained with the procedures adopted here. This does not disagree with the fact that the maser is not really an independent entity; explicit maser dynamics can be actually removed from the set (1)-(4) through a canonical transformation. To conclude, function $L$, defined as it was, seems to be a satisfactory quantity to measure the presence of chaotic activity. negative.
FIG. 4. Normalized orbital divergence $L(t)$. Self-consistent, unbunched case $\left(I_{0}=2\right)$ in (a); self-consistent, bunched case $\left(I_{0}=0.001\right)$ in (b); fixed (non-selfconsistent) wave with $I_{0}=2$ in (c).

\section{B. Localizing chaos}

Given that chaos is present in the system, we now wish to determine in which regions of phase space it is stronger. The interest behind this question is the following. If we were considering a typical wave-particle system, chaos would be stronger near the regions where the particles gyrate with the smallest frequencies. Specializing to our case, chaotic activity would be intimately attached to the outermost orbits of Fig. 3-once more we recall that those are close orbits to autoresonance, moving therefore with the smallest gyrofrequencies. Also, if the picture were valid, chaos would gradually reduce as the elliptic points were approached. The question is then: Is this typical distribution of chaotic orbits the one present in our system? We shall see that the answer is

To perform the investigation, we introduce a quantity $L_{j}(t)$ that shall be seen as measuring a local Lyapunov coefficient. It is defined similarly as $L(t)$ in the form

$$
L_{j}(t)=\ln \left[\sqrt{\sum_{s=-N^{\prime}+1}^{N^{\prime}}\left[I_{j+s}(t)-I_{N^{\prime}+s}^{\prime}(t)\right]^{2}+\left[\phi_{j+s}(t)-\phi_{N^{\prime}+s}^{\prime}(t)\right]^{2}}\right]
$$

where $I_{N^{\prime}+s}^{\prime}=I_{j+s}(1+\delta)$ initially, with $\phi^{\prime}$ defined likewise. What this kind of function really does is just to measure an averaged exponential divergence around the orbit described by the "real" particle label $j$. We choose $N^{\prime}$ small with respect to $N$ but large with respect to unity. We typically take $2 N^{\prime} / N \sim 0.1$.

Recalling that $N=500$, let us then plot, in Fig. 5, function $L_{j}$ for four representative cases: $j=1, j=65$, $j=125$, and $j=150$. The first case corresponds to initial gyrophases near $\phi=0$, the second to $\phi=\pi / 4$, the third to $\phi=\pi / 2$, and the last to $\phi=3 \pi / 5$. Chaos is larger for $j=150$ and $j=65$, and smaller in the other two cases.

Particles in the neighborhood of $j=150$ are those occupying the outermost orbits shown in Fig. 3. They would not follow these very open orbits undergoing the associated large acceleration if the wave were kept constant. What happens, however, is that the complicated nonlinear wave-particle interaction and the associated self-consistent variation of amplitude and phase of the maser displace the particles towards that special location during the very initial stages of the dynamics. This is what we have observed individually accompanying the orbit of the $j=150$ particle. The same complicated non- 


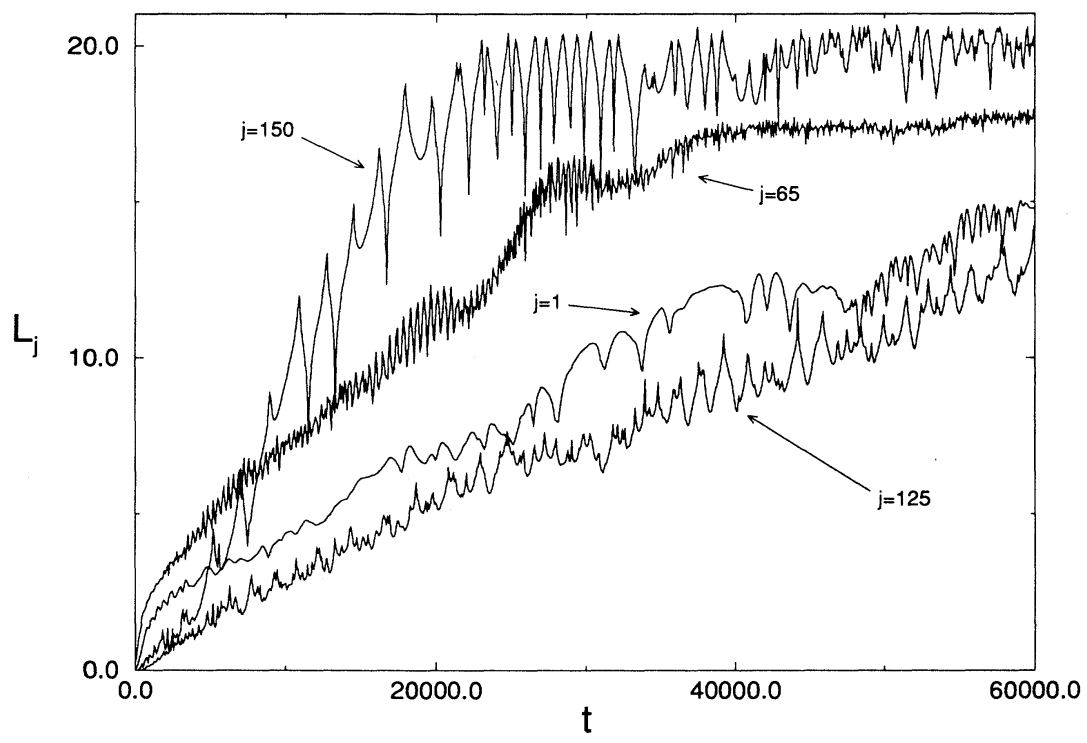

FIG. 5. Normalized local orbital divergence $L_{j}(t)$ for $I_{0}=2$.

linear wave-particle interaction removes the $j=125$ particle from its originally autoresonant position, pushing it into the bulk of the particle distribution-we point out that the resulting action excursion of the $j=125$ particle, when the wave self-consistently varies, is smaller than the action excursion of the $j=150$ particle, for instance. In any case, the orbital period $T_{o r b}$ of particles presenting those large-action excursions is very large compared with the shortest period of the wave fluctuations, $T_{s}$; one has $T_{o r b} \gg T_{s}$. This kind of dynamics thus contains all the aspects of separatrix chaos. In particular, as known from theories describing separatrix chaos [8], the great disparity of time scales represented by the inequality above reduces the distance between neighboring resonances, guaranteeing the presence of resonance overlap and chaos even for the very small amplitude of the fluctuations we observe in the simulations.

Some words are necessary before continuing the investigation. Note that resonance overlap can be obtained for arbitrarily small amplitude fluctuations not only if $T_{\text {orb }}$ is much larger than some typical time scale of the fluctuations. If $T_{\text {orb }}$ is much smaller than typical time scales and if the fluctuations are nonharmonic enough, resonance overlap conditions are again met. The reasoning leading to this conclusion has been developed in Ref. [11] and is just about the same used to explain separatrix chaos. This issue shall be further analyzed in the next section. Here we state the basic results: if $T_{\text {orb }}$ is much larger than typical time scales of the fluctuations, harmonics of the orbital frequency are temporally in resonance with the basic wave frequency (this is separatrix chaos), while if $T_{\text {orb }}$ is much smaller than the typical scales, harmonics of the wave frequency (if the wave is sufficiently nonharmonic) are in resonance with the basic orbital frequency. Thus, in the present case one can expect some relatively intense chaotic activity not only when $T_{\text {orb }} \gg T_{s}$, as in the previous case, but also when $T_{\text {orb }} \ll T_{l}$.

Given these facts, let us then carry on our study. As one penetrates into the bulk with smaller values of $j$ ( $j=$ 125), chaos diminishes. This happens because for this region of the bulk, $T_{\text {orb }}$ appears to be neither sufficiently larger than $T_{s}$ nor sufficiently smaller than $T_{l}$, such that resonance overlap could be as intense as in the former case. Note that chaos is not completely absent; it is only noticeably smaller than previously.

Proceeding further to smaller values of $j(j=65)$ labeling particles which are nearer the elliptic points, one arrives at a situation for which chaos is stronger, again. In that case, one has $T_{\text {orb }} \ll T_{l}$ and chaos is indeed expected to become relatively strong again. Chaotic activity is not typically present at positions close to elliptic fixed points, but here it is as strong as at those positions closer to the separatrix. We emphasize again that its occurrence can be explained in terms of the great disparity between the scales of the relevant frequencies; resonance overlap conditions are again met and chaotic activity is observable once more.

Finally, when one is extremely close to the elliptic fixed point chaos diminishes again. This feature is explainable on basis of the prevailing stability of the elliptic fixed point $[11,14]$. In what follows, by "near" the elliptic points we actually mean not so near that the particles would be captured within its very short stability range.

Note that although chaos is inhomogeneously distributed over the phase space, it is never really absent. We have identified some predominant time scales in the simulations and have used these time scales to explain why chaos is stronger in some regions than in others. The actual maser wave is, however, completely aperiodic and contains larger and smaller scales than the ones we pointed out. In other words, one can say that each particle is actually submitted to the action of an aperiodic drive. Accepting this fact, one can then make use of further results obtained in Ref. [11], which state that if a single action-angle pair of variables interacts with an aperiodic perturbation, no Kolmogorov-Arnold-Moser (KAM) surfaces are formed and chaos, along with free unbounded 
stochastic diffusion, develops everywhere. Rigorously speaking, although the distribution of chaotic orbits is not homogeneous, this ubiquity of chaos is what we have observed.

\section{ARNOLD DIFFUSION AND LOW-DIMENSIONAL MODEL}

Let us recall some points analyzed so far. Chaos is present on the entire phase space. In particular, one observes relatively strong chaos in regions such that $T_{\text {orb }} \sim$ $T_{s} \ll T_{l}$. This is not typical of wave-particle systems where strong chaos is mostly concentrated at the separatrix, with the orbital periods satisfying $T_{\text {orb }} \sim T_{l} \gg T_{s}$. As mentioned, in our particular case this last condition would constrain chaos to the vicinity of the autoresonant orbits.

Now, as mentioned earlier, Boozer [11] recognizes chaotic activity for $T_{\text {orb }} \ll T_{l}$ as an Arnold diffusion process. The point is that if the driving force acting on the particles contains a sufficient amount of nonharmonicity, chaos, and presumably chaotic diffusion, may be present near elliptic points (i.e., for relatively small values of $\left.T_{\text {orb }}\right)$, even for the very small perturbing amplitudes like the ones obtained in the present simulations. Rigorously speaking, Boozer shows that the threshold for resonance overlap vanishes if the perturbing signal is aperiodic. In the calculations Boozer represents the aperiodic signal as a periodic one whose period tends to infinity. Using continuity, one is then lead to conclude that if the period is finite but large, as $T_{l}$ in our case, the threshold for resonance overlap is still very small for orbits satisfying $T_{\text {orb }} \ll T_{l}$ and chaos with small perturbing amplitudes can easily occur. As discussed before we emphasize that inspection of Fig. 3(b) reveals that larger periods than $T_{l}$ may be present in our wave spectrumfrom this perspective one can say that one has in fact
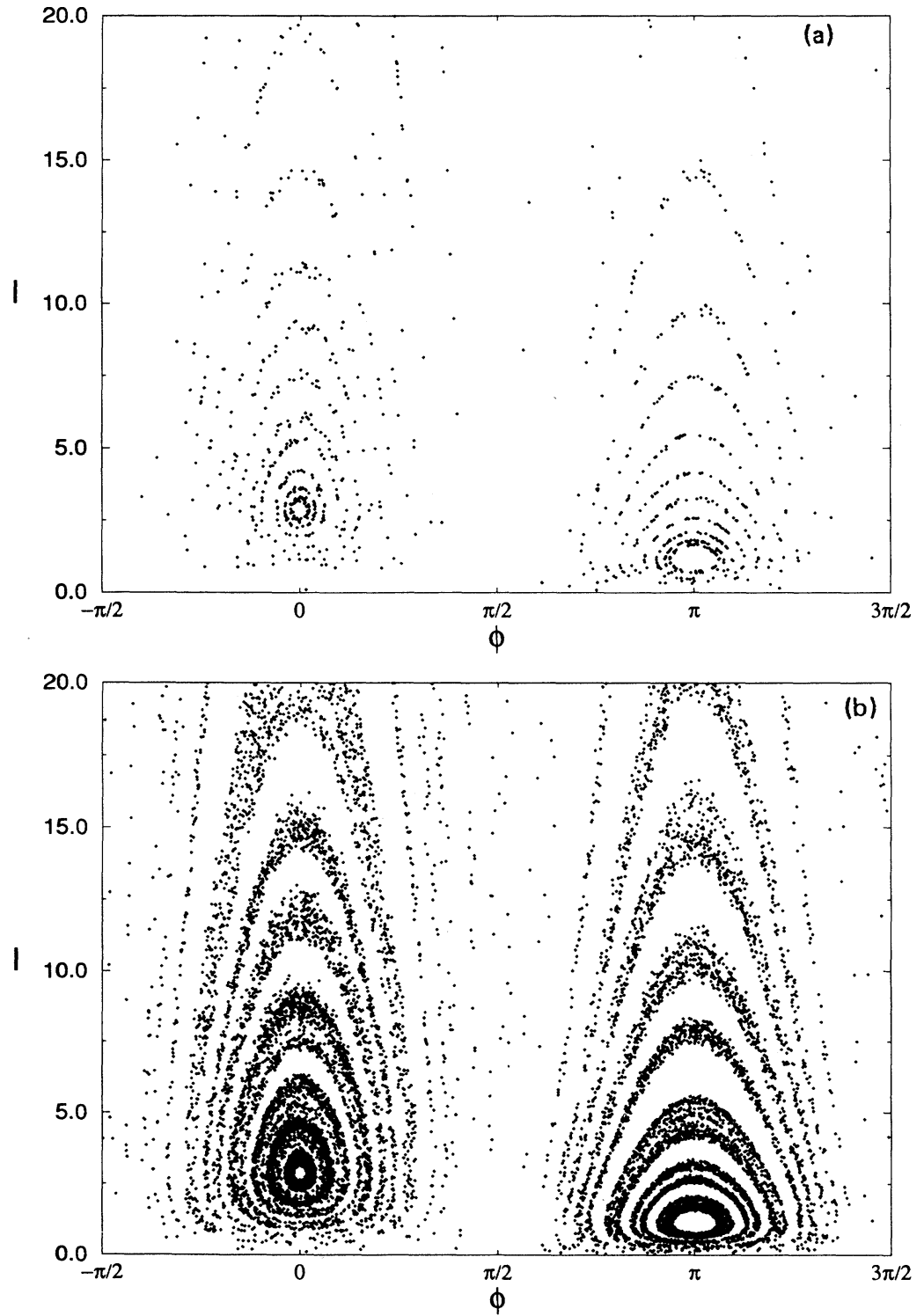

FIG. 6. Chaotic diffusion obtained with the real system for $I_{0}=2 ; t=3.5 \times 10^{4}$ in (a), $t=5 \times 10^{5}$ in (b). 
an aperiodic signal. However, for periods longer than $T_{l}$ the spectral amplitude is small. This is the circumstance that made us focus on orbital periods satisfying $T_{\text {orb }} \ll T_{l}$, where strongest chaotic activity is expected.

We shall not explicitly calculate the diffusion coefficients of the system, but Fig. 6 clearly indicates that diffusion is indeed present. Figure 6(a) is a periodic plot of 30 selected particles (out of the 500 particles used in the simulations) on phase space up to $t=3.5 \times 10^{4}$ and Fig. $6(\mathrm{~b})$ is the same kind of plot up to $t=5 \times 10^{5}$. These plots, constructed on basis of the exact governing Eqs. (1)-(4), can be compared with similar pictures obtainable from a model encompassing the basic characteristics detected.

To build up the model we recall that both phase and amplitude of the wave consist of an average component plus a small fluctuation whose longest time scale results from the interaction with autoresonant particles and whose shortest time scale comes from the interaction with the bulk of the particles distribution. Besides, several numerical simulations have revealed that the wave phase dynamics seems to be more important than amplitude dynamics if one is speaking about chaos. Therefore we choose the following form for our varying maser field:

$$
\rho_{\text {model }}(t)=\text { const }
$$

and

$$
\sigma_{\text {model }}(t)= \begin{cases}+\epsilon & \text { if } n T<t<\left(n+\frac{1}{2}\right) T \\ -\epsilon & \text { if }\left(n+\frac{1}{2}\right) T<t<(n+1) T\end{cases}
$$

where $n=1,2,3, \ldots$ and $\epsilon \ll 1$. Period $T$ is chosen to reflect the actual numerical results. In his analytical calculations, Boozer takes the limit $T \rightarrow \infty$ to represent an aperiodic signal as the limit of a periodic one. In the simulations, we model the aperiodicity taking $T$ to be
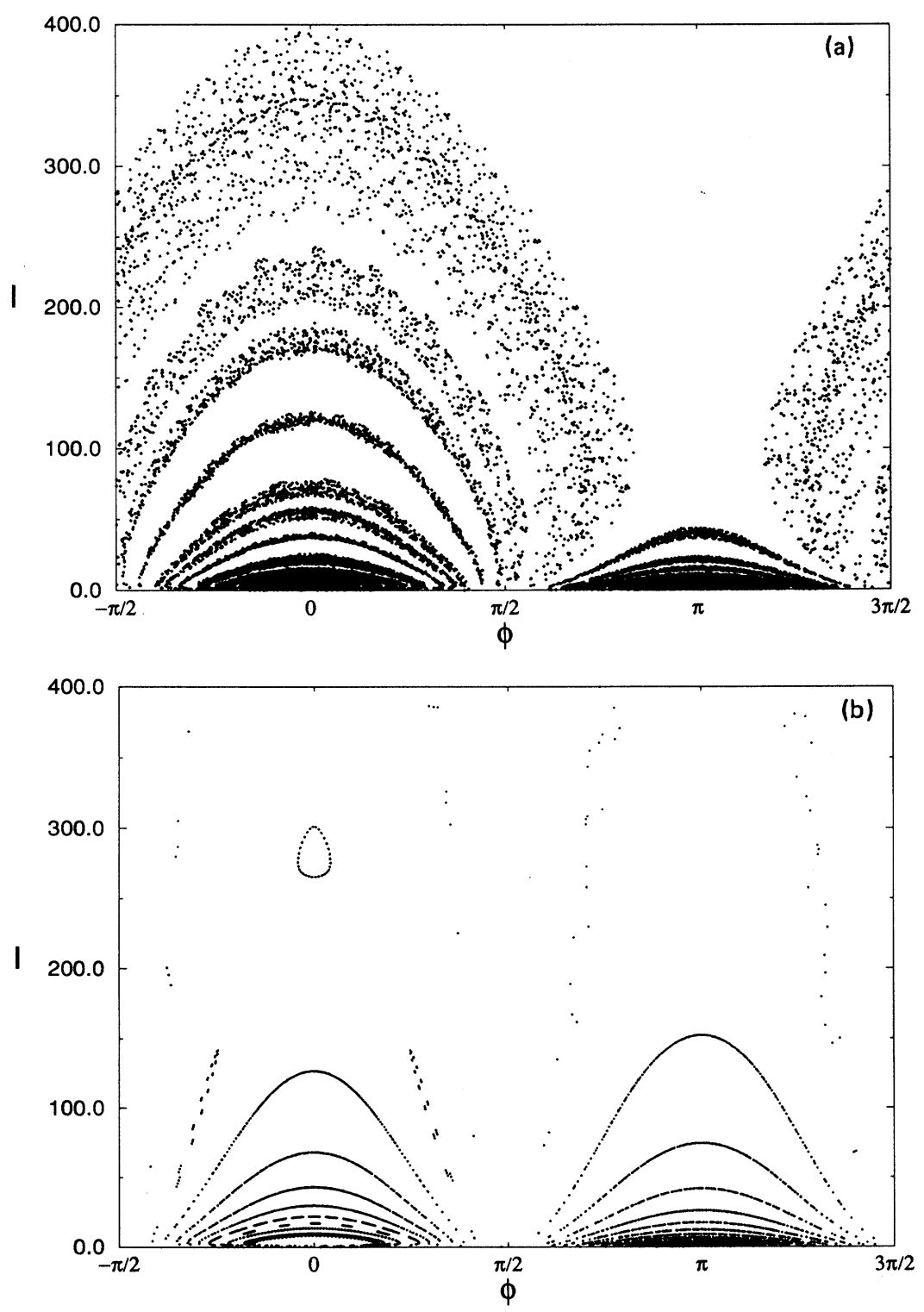

FIG. 7. Chaotic orbits of the real system (a), as compared to the ones obtained with the sine-driven system (b), with a constant wave amplitude $\rho=60$. The period of the sine driver is $T=5000$. 
very large, comparable to the longest period-in practice this means $T \sim 5000$. This should suffice to study chaos near the elliptic points where the periods satisfy $T \gg T_{\text {elliptic }} \sim 10$. The sharp edges of $\sigma_{\text {model }}$ as a function of time provide the shortest time scales which in turn result in the nonharmonic character for the variable. As remarkably found out by Boozer [11], it is precisely the presence of the nonharmonicity in the wave spectrum that is responsible for the profusion of resonance overlaps near elliptic points. In more informal words, for such long periods and high degrees of nonharmonicity, the elliptic points effectively see the signal as an aperiodic one and we can have hope to detect Arnold diffusion developing.

On the other hand, if one had a perturbation with few relevant harmonics only, chaotic activity near the elliptic points would be much smaller, or even absent for the low values of $\epsilon$ we made use of (we have observed that the actual wave phase fluctuations are well represented by $\epsilon=0.01)$. We illustrate this feature, before starting with the study of the kicked model (10), in Fig. 7. In this figure we compare an expanded version of Fig. 6(b) along the $I$ axis, so that one can see the separatrix orbits more clearly [Fig. 7(a)], with the phase space obtained when the maser phase is modeled by a simply harmonically varying $\sigma, \sigma=\epsilon \sin [2 \pi(t / 5000)$ ] [Fig. 7(b)]. Separatrix chaos is seen in both cases, but only in the first does chaos extend to innermost regions of phase space; in the second case the phase space becomes essentially regular as one moves towards the elliptic points, which does not agree with the results of the self-consistent simulations.

Then, we finally make use of the nonharmonic model (10) to study diffusion in Fig. 8, where the time limits used are the same as the ones of Fig. 6, i.e., in Fig. 8(a) the computer run is performed until $t=3.5 \times 10^{4}$ and in Fig. 8(b) until $t=5 \times 10^{5}$.

By comparing Figs. 8(a) and 8(b), one notices that
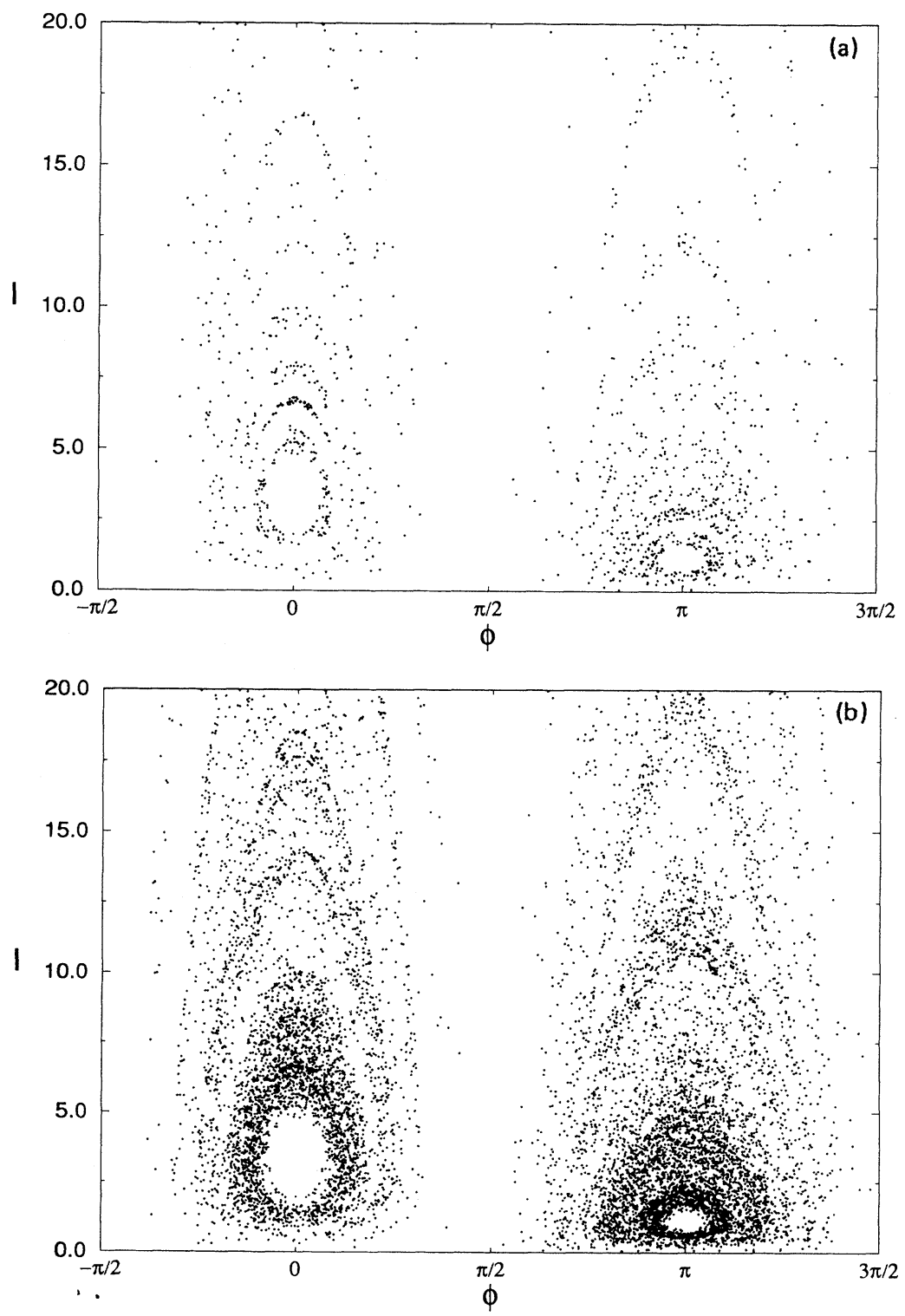

FIG. 8. The same as in Fig. 6, now obtained with the nonharmonic model. The pe$\operatorname{riod}$ is $T=5000$. 
diffusion is indeed present. Moreover, the sudden orbital regularization, as ones gets very close to the elliptic point, can be also appreciated. Note that the modeled wave, although completely regular, appears to well reproduce the simulation results; the nonharmonic character of the model suffices to generate the type of chaos observed.

A question could be raised concerning the accuracy of the use of a driver model with discontinuous derivatives in a set of continuous differential equations. We point out, however, that there is no such problem because the derivatives of the driver are never calculated-only the derivatives of particle variables are necessary. An additional test to check out the validity of the numerical procedures is to represent the periodic step in terms of its analytical trigonometric Fourier series. We did that, gradually increasing the number $N$ of terms in the series to see that while for $N \sim 1$ chaos is far from the elliptic point [this would be similar to the result presented in Fig. 7(b)], for $N \gg 1$ (we took $N=1000$ ) chaos extends to the innermost regions of the phase space, just as in the case of the step driver whose related phase space is represented in Fig. 8.

\section{FINAL REMARKS}

In this paper we have studied the nonlinear dynamics of a cyclotron-maser accelerator as a function of the initial beam action $I_{0}$. It is found that while for $I_{0} \ll 1$ bunching mechanisms regularize the overall dynamics, for $I_{0} \sim 1$ the dynamics is essentially chaotic.

In the latter case, we have localized the strongest chaotic regions on the appropriate phase space. Chaos is found to be located not only close to the autoresonance corresponding to the separatrix motion, but also near the elliptic fixed points, which are not typical locations for chaotic activity. This latter feature can be understood on basis of the great disparity between the orbital period and the longest time scale of the maser field fluctuations; as in the separatrix case, it is possible to show that this disparity dramatically reduces the threshold for resonance overlap [11].

\section{ACKNOWLEDGMENTS}

This work was partially supported by Financiadora de Estudos e Projetos (FINEP) and Conselho Nacional de Desenvolvimento Científico e Tecnológico ( $\mathrm{CNPq}$ ), Brazil. Numerical simulations were performed on the CRAY YMP-2E computer at the Universidade Federal do Rio Grande do Sul Supercomputing CenterCESUP/UFRGS.
[1] P. Sprangle, L. Vlahos, and C.M. Tang, IEEE Trans. Nucl. Sci. NS-30, 3177 (1983).

[2] C.S. Roberts and S.J. Buchsbaum, Phys. Rev. 135, 381 (1964).

[3] S.P. Kuo and G. Schmidt, J. Appl. Phys. 58, 3646 (1985).

[4] R. Shpitalnik, C. Cohen, F. Dothan, and L. Friedland, J. Appl. Phys. 70, 1101 (1991).

[5] C. Wang and J.L. Hirshfield, Phys. Rev. E 51, 2456 (1995).

[6] C. Chen, Phys. Rev. A 46, 6654 (1992).

[7] R. Pakter, R. Schneider, and F.B. Rizzato, Phys. Rev. E 47, 3787 (1993); 49, 1594 (1994).
[8] A.J. Lichtenberg and M.A. Lieberman, Regular and Stochastic Motion (Springer, New York, 1983).

[9] R. Pakter, G. Corso, T.S. Caetano, D. Dillenburg, and F.B. Rizzato, Phys. Plasmas 1, 4099 (1994).

[10] A.C.-L. Chian and C.F. Kennel, Astrophys. Space Sci. 97, 9 (1983).

[11] A.H. Boozer, Phys. Lett. A 185, 423 (1994).

[12] B. Hafizi, P. Sprangle, and J.L. Hirshfield, Phys. Rev. E 50, 3077 (1994).

[13] A. Wolf, J.B. Swift, H.L. Swinney, and J. Vastano, Physica D 16, 285 (1985).

[14] G. Corso and F.B. Rizzato, Physica D 80, 296 (1995). 